\title{
Infant search errors: Stage of concept development or stage of memory development
}

\author{
ELIZABETH LIGON BJORK \\ University of California, Los Angeles, California \\ and \\ E. MARK CUMMINGS \\ National Institute of Mental Health, Washington, D. C.
}

\begin{abstract}
An 8-to-12-month-old infant, having found an object hidden at a first location (A), will frequently continue to search at the A location when the object is moved, in full view of the infant, to a second location (B) and hidden there. In Piaget's (1954) theory of the way in which children acquire knowledge of the external world through actions, the occurrence of such $A \bar{B}$ (or Stage IV) search errors is considered to provide critical evidence that such infants are egocentrically concerned with their own actions and do not yet appreciate the systematic nature of spatial relationships or the permanence of objects. The present research, however, casts serious doubt upon the considerable theoretical significance that has been attached to the $A \bar{B}$ error by demonstrating that it occurs primarily as an artifact of the almost universally employed two-choice hiding task, which constrains all search errors made during $B$-hiding trials to be $A \bar{B}$ errors. In two experiments using less constrained hiding tasks, infants demonstrated no tendency to search incorrectly at the A location during B-hiding trials, and they produced a pattern of errors supportive of the notion that search errors result from a memory problem rather than from a con. ceptual one. A memory explanation is proposed to account for the present results as well as for search errors made throughout the sensorimotor period of development.
\end{abstract}

In nearly every area of psychology, the errors subjects make when performing different types of tasks are used to make inferences concerning possible underlying processes or structures. In the study of infants, the errors they make while searching for hidden objects have served - since Piaget's (1954) original observationsas a rich data source for making inferences about the cognitive development of children.

One of the more intriguing and better known errors studied by Piaget (1954) and others is the so-called

Authorship in the present paper is equally shared. The research reported was supported by Research Committee Grants 4-574069-09528-7 and 4-574069-19900-7 from the University of California, Los Angeles. The paper was presented in part at the meeting of the Psychonomic Society, Phoenix, Arizona, November 1979.

The authors are indebted to Janet Matsunaga and Cheryl Maisel for their assistance in the collection of data and to three colleagues, Patricia Greenfield, Alice Healy, and Lynne Reder, for their support and encouragement during this research. The authors also wish to thank Daniel Schacter and two anonymous reviewers for many perceptive and valuable comments leading to numerous improvements in the final version of the present paper. Requests for reprints can be sent to either Elizabeth L. Bjork, Department of Psychology, University of California, Los Angeles, California 90024, or to E. Mark Cummings, Laboratory of Developmental Psychology, National Institute of Mental Health, Building 15K, Bethesda, Maryland 20205.

-John W. Hagen served as Guest Editor for this manuscript.
"A, not B" error, made by infants between the ages of 8 and 12 months. Around this age, infants become capable of finding a completely hidden object when it is hidden in one place, called the A location. However, when the object is then moved, in full view of the infant, to a second location, called $B$, and hidden there, infants frequently err by continuing to search for the object at location $A$-thus, the "A, not B" (or $A \bar{B}$ ) search error.

The $\mathrm{AB}$ error is well documented (Gratch \& Landers, 1971; Piaget, 1954) and has been studied extensively (e.g., Bremner, 1978a, 1978b; Bremner \& Bryant, 1977; Butterworth, 1975, 1976, 1977; Corter, Zucker, \& Galligan, 1980; Evans \& Gratch, 1972; Frye, 1980; Gratch, Appel, Evans, LeCompte, \& Wright, 1974; Harris, 1973, 1974; Schuberth, Werner, \& Lipsitt, 1978). The considerable attention given to the $A \bar{B}$ error occurs, in part, simply because of its intriguing nature, but primarily because of the critical role it plays in Piaget's (1954) theory of the way in which the child acquires knowledge of the external world through action.

In Piaget's (1954) theory, the occurrence of the $A \bar{B}$ error is a critical indicator that an infant is in Stage IV of the sensorimotor period of development. Indeed, the $A \bar{B}$ error frequently is referred to in the literature as "the Stage IV error," as "Piaget's Stage IV error," or as "Piaget's Stage IV object concept error," and the hiding task in which it has been observed, as "Piaget's Stage IV 
task." (See, for example, Bremner, 1978b, Butterworth, 1975, 1977, Evans \& Gratch, 1972, and Gratch et al., 1974.) Stage IV is of particular importance because of its pivotal nature. In previous stages, infants are assumed not to have an awareness of the permanence of objects in space; that is, infants do not conceive of an object as something that continues to exist when it is out of sight. In Stage IV, however, external objects begin for the first time to have a permanence of their own for the infant-as testified by the infant's ability to find them when they are completely hidden in one location.

Even in Stage IV, however, the permanence of objects is assumed to remain closely tied to the infant's actions. When an object, successfully found in one location (A), is moved in full view of the infant from $A$ to a new location (B) and is hidden there, the infant does not search for the object at $B$, but returns to $A$, where he or she has previously found or acted upon the object. That is, the object's existence and position in space are linked to or are partially defined by the infant's action; in a sense, the object exists for the infant as "the object that I find at location A." Thus, when the object is hidden or disappears from view at location $B$, the infant does not search for it at B but at A, since for the infant the object remains "at disposal in the place where the action has made use of it" (Piaget, 1954, p. 50). In Piaget's theory, then, the Stage IV infant's cognitions concerning space and objects are quite different from those of older individuals or adults; and, the $A \bar{B}$ error is regarded as critical evidence in support of this somewhat radical, but widely held, view of the infant's conception of objects and space.

Given the critical role played by the $A \bar{B}$ error in Piaget's (1954) theory of how the child acquires knowledge of the external world of objects and space, it is not surprising that this error has been extensively studied. What is surprising, however, is that investigators have not asked the most fundamental question regarding this behavior: namely, whether 8-to-12-month-old infants do, in fact, reliably search at the A location when erring during B-hiding trials. Research since Piaget has not adequately addressed this question because investigators studying the Stage IV error have, without exception, provided the infant with only two possible search locations (A and B) during B-hiding trials. ${ }^{1}$ Given the constraints of this two-choice situation, if an overt error is made during a B-hiding trial, it must be a returnto-A error. There is no other location in which the infant can incorrectly search. Thus, a primary goal of the present research was to provide a satisfactory test of the occurrence of the $\mathrm{AB}$ error by presenting infants with at least one alternative location to $A$ in which they can incorrectly search when the object is hidden at a second location (B).

A second purpose of the present research was to test whether the previous well-documented occurrence of the $\bar{A} \bar{B}$ error in the two-choice situation might not stem from a memory problem rather than from a conceptual one. That is, incorrect searches at the A location could occur in the two-choice situation-not because the A location has a special significance to the infant, as assumed in Piaget's (1954) action-object interpretation of this error, but because on some B trials, infants have trouble remembering the object's new location and thus incorrectly search at $\mathrm{A}$. In this view of infant search behavior, then, it is assumed that search errors arise not because the infant cannot conceptually understand the fundamental nature of objects and space, but, rather, because the infant cannot always precisely encode, store, or retrieve the currently correct location of an object as it is successively hidden in different spatial locations. If search errors largely stem from such memory problems, one might expect the infant's errors to reflect partial knowledge or retention of the object's new location. For example, in a situation in which there are more than two possible hiding locations, search errors might be expected to cluster around the object's currently correct hiding location or to form a spatial gradient stemming outward from the correct location.

An adequate answer to the first question raised above-whether 8-to-12-month-old infants will reliably make the $A \bar{B}$ error outside the constraints of a twochoice hiding task-requires presenting infants with only one additional hiding location. However, to address our second question adequately-whether $\bar{A} \bar{B}$ errors might stem from a memory problem rather than from a conceptual one-it is necessary to present more than three hiding locations. With only three hiding locations, it would not be possible to test the memory hypothesis's prediction of a spatial gradient of search errors stemming outward from the correct location. Thus, in order to address these two questions simultaneously, infants were presented with a Stage IV hiding task employing five possible hiding locations.

Two further theoretical points should be noted before the method of our first experiment is explained. First, Piaget (1954) did not specifically constrain infant search to A and B locations during B-hiding trials, but it is not clear that Piaget's infants understood that another legitimate search location existed during B-hiding trials. Second, whereas Piaget also considered the possibility that the $\overline{A B}$ error could occur due to memory failure or forgetting, he rejected this possibility in favor of the action-object explanation of the $A \bar{B}$ error. In fact, Piaget's interpretation of the $A \bar{B}$ error assumes that infants do not register or encode the new location (B) in which the object is hidden following their successful finding of it at the old location (A). Thus, although memory is involved in producing the $A \bar{B}$ error in Piaget's theory, the role of memory conceived by Piaget is very different from the role of memory being proposed here. In Piaget's theory, there is a failure of memory in that the new location is not registered by the infant; consequently, such information cannot in any way affect the infant's choice of search locations on B-hiding trials. In contrast, according to the role of memory we are sug- 
gesting, one would expect many of the infant's incorrect searches to reflect partial knowledge or retention of the object's new location.

\section{EXPERIMENT 1}

\section{Method}

\section{Subjects}

The analyses for this study are based on the performance of 36 infants (18 males and 18 females), ranging in age from 8 months 10 days to 11 months 5 days, with a median age of 9 months 11 days. In terms of a frequency distribution of ages, there were 98 -month-old infants, 21 9-month-old infants, 510 -month-old infants, and 111 -month-old infant. The infants were located through birth announcements in a local newspaper, such announcements being automatically published whenever a birth certificate is issued.

In addition to the above infants, six infants $(3$ males and 3 females; median age 9 months 12 days; age range from 8 months 25 days to 11 months 1 day; and located in the same manner) were tested in the present study but excluded from the data analyses. Four of the six infants were excluded because they were incorrect on all A trials (the data from such infants cannot be interpreted with respect to Piaget's (1954) prediction of $\mathrm{A} \overline{\mathrm{B}}$ errors, since for the $\mathrm{A}$ location to acquire a special significance for the infant, the infant must act upon the object at least once at the A location). An additional two infants had to be excluded because they became too upset to continue in the experiment.

\section{Apparatus}

A block of white foam rubber, 30 in. $(76 \mathrm{~cm})$ long, 12 in. $(30 \mathrm{~cm})$ wide, and 4 in. $(10 \mathrm{~cm})$ thick, served as the basic apparatus. Five holes, 5 in. $(12.7 \mathrm{~cm})$ high, 3 in. $(7.62 \mathrm{~cm})$ across, and $1.5 \mathrm{in} .(3.81 \mathrm{~cm})$ deep, were cut into the foam-rubber block. Each hole was $4.5 \mathrm{in} .(11.43 \mathrm{~cm})$ from the hole next to it, measured from center to center, or $1.5 \mathrm{in} .(3.81 \mathrm{~cm})$ from the hole next to it, measured from the two closest edges. Of these five holes, only the far left and far right holes were used as hiding locations, and these were 18 in. $(45.72 \mathrm{~cm})$ apart from center to center. Blue-felt pieces, slightly less than 3.75 in. $(9.5$ $\mathrm{cm})$ wide and 8.75 in. $(22.22 \mathrm{~cm})$ long, were used as hiding covers. With the blue hiding covers in place, adjacent covers were separated by an intervening space of the white foam-rubber apparatus of slightly more than 1 in.

A red plastic key or a red plastic octopus were used as search objects during warm-up trials. During all of the experimental trials for a given subject, a single small rubber animal (a yellow duck or a blue bear) or a small red and yellow plastic rattle was employed as the hiding object. The rubber animal could be squeaked, and the rattle, which contained a single bell, could be shaken to attract the infant's attention before being hidden, but neither type of toy made any noise when moved toward or lowered into the hiding hole. Both types of toys fit entirely within the hiding hole so that the felt cover hiding the toy would lie flat on the apparatus.

\section{Design and Procedure}

The subjects were tested in their own homes on any convenient rug-covered floor. The infants were positioned directly in front of the middle hole of the apparatus. The infant's mother sat directly behind the infant on the floor, and the experimenter sat across from the infant on the opposite side of the apparatus. A second adult recorded the infant's responses and timed the delay from when the toy was hidden until the infant was allowed to search for it.

The infants were given four warm-up trials to familiarize them with the apparatus and with the task of retrieving toys from the apparatus. In two trials, the infant found a toy un- covered, and in two trials, the infant found a toy partially covered at the A location. All infants were able to perform the warm-up trials successfully.

Following the warm-up trials, the infants were given four experimental trials in which the toy was always completely hidden. The experimental trials began with the hiding of the toy two consecutive times at a first location (A). These trials were followed by two consecutive hidings of the same toy at a second location (B). Only the far left and the far right holes served as hiding locations, and each was assigned to be the A or B location equally often. Furthermore, the infants were assigned at random to one of the two possible A-B hiding sequences: far left-far right or far right-far left.

During experimental trials, the experimenter shook or squeaked the toy, while holding it at a point just behind and above the middle hole of the apparatus, until the infant looked at the toy. The toy was then moved to the appropriate hiding location for that trial, lowered into the hole, and covered. This procedure was repeated if the infant stopped looking at the toy before it was covered. The infant was required to wait $3 \mathrm{sec}$ after the toy had been covered before searching for the toy. If the infant tricd to reach for the toy before $3 \mathrm{sec}$ had elapsed, the mother would restrain the infant by gently holding his or her shoulders. After successfully finding the toy, the infant was allowed to play with it for a few seconds before the next trial was begun. If the infant searched unsuccessfully for the toy, the experimenter retrieved the toy for the infant before the infant could search further. The infant was then allowed to play with the toy for a few seconds before the start of the next trial.

\section{Results and Discussion}

In the present section, results are presented in the following general order: first, the overall correct search performance obtained across A and B trials; second, the analyses of search errors relevant to the prediction from Piaget's (1954) action-object account of infant search behavior; and finally, the analyses of errors relevant to the memory explanation's expectation of how search errors should be distributed.

\section{Overall Correct Search Performance}

A trials. As a whole, infants were clearly able to search correctly for the hidden object on A-hiding trials. On the first A trial, 30 of the 36 infants (or 83\%) searched for the object in its correct location, and on the second A trial, 31 of the 36 infants (or $86 \%$ ) searched for the object in its correct location. Over the two A trials, 25 infants made no errors, and all 36 infants were correct on at least one A trial.

B trials. On the first B-hiding trial, only 18 of the 36 infants (or 50\%) correctly searched for the hidden object. On the second B-hiding trial, 25 infants searched correctly for the hidden object, with 10 infants making search errors and 1 infant failing to search. The drop in performance between the last A-hiding trial $(86 \%)$ and the first B-hiding trial $(50 \%)$ is significant by a sign test $(z=2.92, p<.002)$.

\section{Action-Object Prediction}

With respect to the fundamental question of whether 8-12-month-old infants will reliably make the $A \bar{B}$ error outside the constraints of the two-choice situation-in accordance with Piaget's (1954) action-object theory-the 
relevant data are as follows: On the first B-hiding trial, 18 search errors were made; however, only 1 of these errors was an A-returning error. All other incorrect searches occurred at locations in which the object had never been hidden and, thus, never acted upon.

On the second B-hiding trial, the pattern remained the same. Of the 10 overt search errors made, only 1 was an A-returning error, the remaining 9 being incorrect searches occurring at locations in which the object had never been hidden or acted upon by the infant.

In addition, analyses limited to only those infants who were correct on at least the last A-hiding trial or those infants who were correct on both A-hiding trials show the same pattern of results. There are 31 infants who qualified for the first set; of these, 16 searched correctly on the first B-hiding trial, 14 incorrectly searched in a location in which the object had never been hidden, and 1 returned to the A location to search for the hidden object. On the second B-hiding trial, 21 of these infants searched correctly, 9 incorrectly searched in a location in which the object had never been hidden, and 1 returned to the $A$ location to search for the hidden object. Similarly, for the subset of 25 infants who were correct on both A trials, 16 searched correctly on the first B trial, 8 incorrectly searched in a location in which the object had never been hidden, and 1 returned to the A location to search for the hidden object. On the second B trial, 18 of these infants searched correctly, 6 incorrectly searched in a location in which the object had never been hidden, and 1 returned to the A location to search. In short, whether one looks at the behavior of all infants in the present experiment, or at the subset of infants who were correct on at least the last A-hiding trial, or only at those infants who were correct on both A-hiding trials, the pattern of results is the same.

In addition to showing no tendency to make Areturning search errors on B-hiding trials, the infants also showed no tendency to search at locations close to the A location when they erred during B-hiding trials. To illustrate, of the 18 search errors made on the first B trial, only 3 errors involved search at the A location or the hole closest to the A location; and on the second B trial, only 2 of the 10 overt search errors made involved search at the A location or the hole closest to the A location. In fact, across the two B-hiding trials, significantly more search errors occurred in the incorrect location farthest from the A location than occurred in the location closest to the A location $(\mathrm{p}<.01$, binomial test).

Clearly, the patterns of errors obtained on both the first and second B-hiding trials of this experiment are inconsistent with the predictions of Piaget's (1954) action-object theory. In an unconstrained Stage IV hiding task, the infants demonstrated no tendency to return to the $A$ location on $B$-hiding trials. This finding indicates that the $A \bar{B}$ error observed in previous Stage IV hiding tasks has occurred primarily as an artifact of providing the infant with only two clear search alterna- tives (A and B) during B-hiding trials. When the infants were not constrained by the experimental paradigm to return to the $\mathrm{A}$ location when they erred during B-hiding trials, the A location appeared to hold no special significance for the 8-to-12-month-old infants, in direct contradiction of Piaget's contention that at this stage of development the object conceptually remains at disposal in the location in which the infant's action has made use of it.

\section{Memory Prediction}

In order to evaluate the proposed role of memory in the production of infant search errors, it is necessary to analyze where infants search in relation to the actual location of the hidden object on any given trial. Such an analysis is presented in Table 1, which shows the number of search errors made at each possible incorrect location as a function of its distance from the correct location on each A- and B-hiding trial.

As can be seen in the top half of Table 1, most infants searched either at or close to the correct hiding location on the first A-hiding trial, with this tendency increasing on the second A-hiding trial.

However, of more concern to the present memory hypothesis are the B-trial data shown in the bottom half of Table 1. There it can be seen that the prediction of a spatial gradient of errors stemming outward from the currently correct location of the object is rather dramatically upheld on the first B-hiding trial. On the second B-hiding trial, fewer incorrect searches occur, making a distribution analysis somewhat tentative. Nonetheless, the most frequent location of incorrect searches remains the location closest to the currently correct location of the hidden object.

It should be noted that in the context of the present experiment, the action-object hypothesis and the memory hypothesis make essentially opposite predictions concerning the direction of search attempts that should be observed in the present hiding task. The action-object hypothesis predicts that incorrect search attempts

Table 1

Frequency of Search at the Five Alternative Locations During the A and B Trials of Experiment 1

\begin{tabular}{|c|c|c|c|c|c|c|}
\hline \multirow{3}{*}{$\begin{array}{c}\text { Trial } \\
\text { Number }\end{array}$} & \multirow{3}{*}{$\begin{array}{c}\text { Correct } \\
\text { Hole }\end{array}$} & \multicolumn{4}{|c|}{ Search Location } & \multirow{3}{*}{$\begin{array}{c}\text { Failures to } \\
\text { Search }\end{array}$} \\
\hline & & \multicolumn{4}{|c|}{ Closest Hole } & \\
\hline & & $1 \mathrm{st}$ & 2nd & 3 rd & 4th & \\
\hline \multicolumn{7}{|c|}{ A Trials } \\
\hline 1 & 30 & 4 & 2 & 0 & 0 & 0 \\
\hline 2 & 31 & 5 & 0 & 0 & 0 & 0 \\
\hline \multicolumn{7}{|c|}{ B Trials } \\
\hline 1 & 18 & 8 & 7 & 2 & 1 & 0 \\
\hline 2 & 25 & 7 & 1 & 1 & 1 & 1 \\
\hline
\end{tabular}

Note-During $B$ trials, the 4th closest hole is the $A$ location. Maximum number of search attempts possible at any one loca. tion $=36$. 
should be located at or near the A location on B-hiding trials; in contrast, the memory hypothesis predicts that search attempts should occur at or near the object's current location even on B-hiding trials. To test which of these predictions was correct, as well as the overall direction of search tendencies, the number of searches made at the B location and the location closest to the $B$ location was compared with the number of searches made at the $A$ location and the location closest to the A location during both the first and second B-hiding trials. (Searches at the middle hole were omitted from these analyses because it was not clear whether middlehole searches indicated a tendency to search in the direction of the correct $B$ location or in the direction of the A location.) These comparisons revealed no tendency for infants to search at or near the A location; instead, they revealed a significant tendency for infants to search at or near the currently correct location during both the first and second B-hiding trials $(z=4.56, p<.001$, and $z=4.94, p<.001$, respectively).

Thus, on both A and B trials, the distributions of search errors appear to reflect partial knowledge or retention of the current spatial location of the object, consistent with the assumptions of the present memory explanation of infant search behavior. We also feel that the significant drop in performance from the last $\mathrm{A}$ hiding trial to the first B-hiding trial, as well as the poorer performance on the first $B$ trial than on the first A trial, are consistent with the present proposal that infant search errors are primarily produced by a memory limitation rather than by a conceptual one. These aspects of the results are discussed in the General Discussion section following Experiment 2.

\section{EXPERIMENT 2}

Proponents of an object-action account of infant search behavior might want to argue that, because the infants in Experiment 1 did not make the $A \bar{B}$ error, they were not in what Piaget (1954) referred to as Stage IV, but were perhaps in some later stage in which object and action are no longer tied together so egocentrically as to produce the $A \bar{B}$ or Stage IV error.

There are several problems with this argument. First, although we realize that Stage IV is not defined as a particular age, but rather as a level of cognitive development, the present infants were of the exact average age ( 9 months) specified by Piaget (1954, p. 45) for the onset of Stage IV. Second, the present infants were of essentially the same mean age and age range as infants in the previous Stage IV studies that are considered to have documented the $\bar{A} \bar{B}$ error (e.g., Butterworth, 1975, 1976; Evans \& Gratch, 1972; Gratch et al., 1974; Harris, 1973). Third, and most importantly, a more advanced, Stage $\mathrm{V}$ infant should theoretically not make any errors on the Stage IV task (Piaget, 1954, pp. 66-67), whereas a majority of the infants in the present experiment made at least one B-trial error. Nevertheless, to dismiss such arguments and to demonstrate unambiguously that the infants of Experiment 1 were at the same level of cognitive development as the infants previously reported in the literature as making the $\mathbf{A B}$ error in twochoice hiding tasks, a second experiment was conducted. In Experiment 2, the same infants were tested for the occurrence of the $A \bar{B}$ error both in the traditional twochoice hiding task of previous Stage IV research and in the five-choice hiding task of Experiment 1. Thus, Experiment 2 allowed us to determine if the same infants who made $\bar{A} \bar{B}$ errors in the traditional twochoice task and who, therefore, would be classified as Stage IV infants in accordance with the criteria of previous literature, would also make $A \bar{B}$ errors in a hiding task that did not constrain B-trial search errors to be returns to the A location.

\section{Method}

\section{Subjects}

The analyses for this study are based on the performance of 16 infants (6 females and 10 males), ranging in age from 8 months 10 days to 10 months 8 days and having a median age of 9 months 5 days. In terms of a frequency distribution of ages, there were 58 -month-old infants, 109 -month-old infants, and 1 10-month-old infant. The infants were located as in Experiment 1 , by means of birth announcements in a local newspaper.

Five other infants (three males and two females; median age 9 months 17 days; age range from 9 months 3 days to 10 months 30 days; located in the same manner) were tested in the present study but were excluded from the data analyses. Three of these infants were excluded because they were incorrect on all $A$ trials. The remaining two infants were excluded because they became too upset to continue in the experiment.

\section{Apparatus}

The apparatus for the five-choice hiding task was identical to that used in Experiment I; and, as before, only the far left and far right holes were used as hiding locations.

The apparatus for the two-choice hiding task was made from a block of white foam rubber identical in size and color to that for the five-choice hiding task. The two hiding holes cut into this block of foam rubber were also identical in dimensions to the holes of the five-choice apparatus, measuring $5 \mathrm{in.}(12.7 \mathrm{~cm})$ high, 3 in. $(7.62 \mathrm{~cm})$ across, and 1.5 in. $(3.81 \mathrm{~cm})$ deep. The two holes were separated by $9.5 \mathrm{in} .(24.13 \mathrm{~cm})$, measured from center to center, or 6.5 in. $(16.51 \mathrm{~cm})$, measured from the two closest edges. Separation between the two hiding locations in previous Stage IV studies has varied widely-for example, 4 in. (Harris, 1973), 7.87 in. (Butterworth, 1975), 11.02 in. (Bremner, 1978a; Bremner \& Bryant, 1977), 12 in. (Evans \& Gratch, 1972; Gratch et al., 1974), and 18 in. (Harris, 1974). Furthermore, these measurements generally have not been reported in such a way as to enable determination of whether they referred to separations measured from centes to center or from the two closest edges of the hiding locations. Thus, the current separation distance was chosen so as to be roughly in the middle of the range of separations previously employed in two-choice hiding tasks.

Blue pieces of felt identical to those employed in Experiment 1 were used as hiding covers for both the two-choice and the five-choice apparatuses. The same red plastic key and red plastic octopus were used as search objects during the warm-up trials; and, during all of the experimental trials for a given subject, either one of the small rubber animals or the small red and yellow plastic rattle of Experiment 1 was employed as the hiding object. 


\section{Design and Procedure}

Most aspects of the testing procedure were the same as in Experiment 1. Infants were again tested in their own homes on any convenient rug-covered floor. They were positioned directly in front of the middle hole of the five-choice apparatus or equidistant between the two holes in the two-choice apparatus. The infant's mother sat directly behind the infant on the floor, and the experimenter sat across from the infant on the opposite side of the apparatus. A second adult recorded the infant's responses and timed the delay interval employed.

The warm-up trials were conducted in the same manner as in Experiment 1, and all infants were able to perform the warmup trials successfully.

The experimental procedure was the same as that of Experiment 1 , except that (1) one-half of the infants were tested first on the two-choice apparatus and then on the five-choice apparatus and one-half were tested first on the five-choice apparatus and then on the two-choice apparatus, and (2) the infants were given 3 A-hiding trials and 3 B-hiding trials in both the twochoice and the five-choice hiding task in order to get a somewhat better look at changes in performance over trials at the same location. (Although 4 or 5 trials at each location would probably have been better for this purpose, pilot work had indicated that 12 total trials was about the maximum number we could hope to conduct without stressing the infants.) As in Experiment 1, the hole used as the A or B location for each type of apparatus was counterbalanced across subjects, and a 3-sec delay was imposed between the time the toy was hidden and the time the infant was allowed to search for it.

\section{Results and Discussion}

In the present section, results are presented in the following general order: first, analyses comparing search performance across the $\mathrm{A}$ and $\mathrm{B}$ trials for the twochoice versus the five-choice hiding task; and second, the analysis of search errors occurring on the five-choice task in relation to the correct location of the hidden object on each A and B trial.

\section{Two-Choice Versus Five-Choice Search Behavior}

A trials. The frequencies of correct searches and types of search errors made during the three A-hiding trials on each type of apparatus are presented in Table 2, in

Table 2

Frequency of Correct Searches and Types of Search Errors on A Trials in the Two-Choice and Five-Choice Tasks of Experiment 2

\begin{tabular}{cccc}
\hline & \multicolumn{3}{c}{ Response Type } \\
\cline { 3 - 4 } Trial & $\begin{array}{c}\text { Correct } \\
\text { Sumber }\end{array}$ & $\begin{array}{c}\text { Incorrect } \\
\text { Searches }\end{array}$ & $\begin{array}{c}\text { Failures to } \\
\text { Search }\end{array}$ \\
\hline \multicolumn{4}{c}{ Two-Choice Hiding Task } \\
1 & 13 & 1 & 2 \\
2 & 15 & 1 & 0 \\
3 & 15 & 1 & 0 \\
& Five-Choice Hiding Task & \\
1 & 10 & 6 & 0 \\
2 & 11 & 5 & 0 \\
3 & 14 & 2 & 0 \\
\hline
\end{tabular}

Note-F denotes locations in which the object had not been hidden for that trial or any preceding hiding trial. Maximum correct on each trial $=16$; chance $=8$ on the two-choice task and 3.2 on the five-choice task. which $\mathrm{F}$ denotes those locations in which the object has never been hidden. In general, infants were clearly able to search correctly for the hidden object on A-hiding trials in the two-choice task, with 13 of the 16 subjects (or $81 \%$ ) searching correctly on the first $\mathrm{A}$ trial and 15 of the 16 subjects (or 94\%) searching correctly on the second and third A trials. Over the three A trials, 12 infants made no errors. Of the remaining four, 3 made one A-trial error and 1 made two A-trial errors. All infants were correct on at least one A-hiding trial.

Initially at least, infants appear to have had slightly more difficulty on the five-choice task, with 10 of the 16 infants (or 63\%) searching correctly on the first A trial and 11 (or $69 \%$ ) searching correctly on the second A trial. However, by the third A trial, 14 (88\%) of the subjects searched correctly. Over the three A-hiding trials, 7 infants managed to make no errors, 5 made one A-trial error, and 4 made two A-trial errors. However, all infants were correct on at least one A-hiding trial.

That infants could have more trouble on the initial A-hiding trials of a five-choice task than on those of a two-choice task is consistent with the present memory hypothesis. However, the present infants' performance on the first and second trials of the five-choice task was also poorer than that of the infants in Experiment 1. Two factors occur to us as possible reasons for this difference. First, the age composition of the two samples was somewhat different, with the Experiment 2 sample being slightly younger as a whole than the Experiment 1 sample. In Experiment $1,17 \%$ of the infants were 10 to 11 months old, as compared with $6 \%$ of the Experiment 2 infants; and, $31 \%$ of the Experiment 2 infants were 8 months old, as compared with $25 \%$ of the Experiment 1 infants. Second, it seems likely that the poorer five-choice performance in Experiment 2 occurred because half of the infants had been tested on a twochoice task prior to being tested on the five-choice task and, thus, were quite likely to be somewhat fatigued. Indeed, $64 \%$ of the errors made on the first and second A-hiding trials were made by infants who performed the five-choice task following the two-choice task.

B trials for all infants. The frequencies of correct searches and types of search errors made during the three B-hiding trials for the two-choice and five-choice hiding tasks are presented in Table 3 , in which $F$ denotes those locations in which the object has never been hidden for that trial or for any preceding hiding trial. First, with respect to performance in the two-choice task, shown in the top half of Table 3 , it is clear that many incorrect searches were made on the B-hiding trials and that these errors occurred at the A location; in short, they were traditional $A \bar{B}$ errors. Across the three B-hiding trials, a total of 14 such errors were made, with 9 occurring on the first B-hiding trial, 3 on the second, and 2 on the third. [It should perhaps be pointed out that the proportion of infants making an $A \bar{B}$ error on the first $B$ trial in the present experiment (.52) is comparable to the proportions previously observed in similar hiding situations: e.g., .58 in the "Same Toy 
Table 3

Frequency of Correct Searches and Types of Search Errors on B Trials in the Two-Choice and Five-Choice Tasks of Experiment 2

\begin{tabular}{|c|c|c|c|c|}
\hline \multirow[b]{2}{*}{$\begin{array}{c}\text { Trial } \\
\text { Number }\end{array}$} & \multirow[b]{2}{*}{$\begin{array}{l}\text { Correct } \\
\text { Searches }\end{array}$} & \multicolumn{2}{|c|}{ Respone Type } & \multirow[b]{2}{*}{$\begin{array}{c}\text { Failures to } \\
\text { Search }\end{array}$} \\
\hline & & $\begin{array}{c}\text { Incorrect } \\
\text { Searches } \\
\text { at A }\end{array}$ & $\begin{array}{c}\text { Incorrect } \\
\text { Searches } \\
\text { at F }\end{array}$ & \\
\hline \multicolumn{5}{|c|}{ Two-Choice Hiding Task } \\
\hline 1 & 5 & 9 & & 2 \\
\hline 2 & 13 & 3 & & 0 \\
\hline 3 & 13 & 2 & & 1 \\
\hline \multicolumn{5}{|c|}{ Five-Choice Hiding Task } \\
\hline 1 & 6 & 1 & 9 & 0 \\
\hline 2 & 8 & 0 & 8 & 0 \\
\hline 3 & 12 & 0 & 3 & 1 \\
\hline
\end{tabular}

Note-F denotes locations in which the object had not been hidden for that trial or any preceding hiding trial. Maximum correct on each trial $=16$; chance $=8$ on the two-choice task and 3.2 on the five-choice task.

Condition" of Evans \& Gratch, 1972, and .52 in the "Object Hidden Condition" of Butterworth, 1977.]

Similarly, many incorrect searches were made on the B-hiding trials in the five-choice hiding task. In total, 21 incorrect searches (and 1 failure to search) occurred over the three B-hiding trials in the five-choice hiding task, with 10 occurring on the first B-hiding trial, 8 on the second, and 3 on the third. However, of these 21 overt search errors, only 1 was a return to the A location.

Furthermore, as in Experiment 1, the drop in performance between the last A-hiding trial and the first B-hiding trial is significant by a sign test $(\mathrm{z}=2.47$, $\mathrm{p}<.01$ ).

$B$ trials for infants making $A \bar{B}$ errors in the twochoice task. With respect to the primary question of Experiment 2, the most critical analysis involves only those infants who made at least one $\mathrm{A} \overline{\mathrm{B}}$ error in the twochoice hiding task and who, thus, would be Stage IV infants in accordance with previous research. In the present experiment, there were 10 infants making at least one $\bar{A} \bar{B}$ error in the two-choice task, with 9 of these infants making an $\overline{A B}$ error on the first $B$ trial. These same infants made a total of 15 B-trial errors in the five-choice task. Of these 15 errors, none was a return to the A location.

Thus, the answer to the primary question asked by Experiment 2 seems quite clear: The same infants who made the $A \bar{B}$ error on the traditional two-choice hiding task and who, therefore, would be classified as Stage IV infants according to the criteria used in previous Stage IV research, did not do so in a hiding task involving more than two locations, that is, in a task that did not by its very nature constrain any overt search errors made during $\mathrm{B}$-hiding trials to be $\mathrm{A} \overline{\mathrm{B}}$ errors. Although these infants made many $B$-trial errors on the five-choice task, they did not tend to be returns to the A location as would be predicted by the Piagetian action-object theory. The implications of these results seem unequivocal. The $\mathrm{A} \overline{\mathrm{B}}$ error, taken to indicate Stage IV of cognitive development in Piaget's (1954) theory of object-concept development, occurs primarily as an artifact of the previously employed two-choice hiding task, and, therefore, all theoretical conclusions based upon its occurrence are subject to question.

\section{Distribution of Incorrect Searches}

Although the main purpose of Experiment 2 was to ascertain whether the same infants who made $A \bar{B}$ errors in a two-choice task would also do so in a fivechoice task, the five-choice data obtained in Experiment 2 can also be subjected to the same distance analysis used in Experiment 1 as a further test of the memory explanation of infant search behavior. The results of such an analysis are presented in Table 4, which shows the number of search errors made at each possible incorrect location as a function of its distance from the correct location on each A- and B-hiding trial.

The A-trial data, located in the top half of Table 4, show that, as in Experiment 1, most infants searched either at the correct hiding location or in the location next to it, with the tendency to search at the correct location steadily increasing over trials.

The B-trial data, shown in the bottom half of Table 4, also reveal the same pattern found in Experiment 1. On all three B-hiding trials, errors tend to form a spatial gradient stemming outward from the correct location of the object. On each trial, the majority of errors occur in the location closest to the correct location; and, across trials, search attempts can be seen to converge upon the correct location.

To test for the overall direction of search tendencies, the number of searches made at the B location and the location closest to the B location was compared with the number of searches made at the $A$ location and the location closest to it across the three B-hiding trials. These comparisons revealed a significant tendency for

Table 4

Frequency of Search at the Five Alternative Locations During the $A$ and $B$ Trials of Experiment 2

\begin{tabular}{|c|c|c|c|c|c|c|}
\hline \multirow{3}{*}{$\begin{array}{c}\text { Trial } \\
\text { Number }\end{array}$} & \multirow{3}{*}{$\begin{array}{c}\text { Correct } \\
\text { Hole }\end{array}$} & \multicolumn{4}{|c|}{ Search Location } & \multirow{3}{*}{$\begin{array}{c}\text { Failures to } \\
\text { Search }\end{array}$} \\
\hline & & \multicolumn{4}{|c|}{ Closest Hole } & \\
\hline & & $1 \mathrm{st}$ & 2nd & $3 \mathrm{rd}$ & 4 th & \\
\hline \multicolumn{7}{|c|}{ A Trials } \\
\hline 1 & 10 & 4 & 2 & 0 & 0 & 0 \\
\hline 2 & 11 & 4 & 1 & 0 & 0 & 0 \\
\hline 3 & 14 & 2 & 0 & 0 & 0 & 0 \\
\hline \multicolumn{7}{|c|}{ B Trials } \\
\hline 1 & 6 & 7 & 2 & 0 & 1 & 0 \\
\hline 2 & 8 & 7 & 1 & 0 & 0 & 0 \\
\hline 3 & 12 & 2 & 1 & 0 & 0 & 1 \\
\hline
\end{tabular}

Note-Maximum number of search attempts possible at any one location $=16$. 
infants to search at or near the currently correct location during all three B-hiding trials $(\mathrm{z}=2.73, \mathrm{p}<.005$, $z=3.76, p<.001$, and $z=3.31, p<.001$, for the first, second, and third $B$ trials, respectively).

Furthermore, if one considers only those infants who made at least one $A \bar{B}$ error in the two-choice task, the same trend is clearly present, with more infants searching at or near the correct location on each $B$ trial of the five-choice task than at or near the A location $(\mathrm{z}=2.59$, $\mathrm{p}<.005, z=2.65, \mathrm{p}<.005$, and $z=2.65, \mathrm{p}<.005$, respectively).

Finally, the higher frequency of A-trial errors occurring in the present study makes it possible to compare the types of errors occurring on A versus B trials. It seems clear that there is no qualitative difference between them; both $\mathrm{A}$ and $\mathrm{B}$ search errors tend to cluster around the currently correct location and to converge upon the correct location over trials, a result consistent with the current memory account of infant search behavior. In terms of the present memory account, the demands placed upon the information-processing skills of the infant by $\mathrm{A}$ and B trials are different in degree but not in kind, and, thus, the operation of the same underlying processes should determine the infant's performance on both types of trials. Differences between A- and B-trial performance will be further developed in the General Discussion section below.

Thus, Experiment 2 replicated the findings of Experiment 1 with respect to the location of incorrect search attempts on B-hiding trials. In addition, the A-trial errors obtained show the same distribution pattern as the B-trial errors. Hence, the present results both reconfirm and add new support to the proposed memory account of infant search behavior.

\section{GENERAL DISCUSSION}

Piaget's (1954) interpretation of the search behavior of 8-to-12-month-old infants as perseverative and, thus, indicative of a state of development in which the infant is unable to separate completely action from object has been called into question by the results of the present experiments. Although accorded great theoretical significance, the perseverative or $\mathrm{AB}$ search errors observed by Piaget and many others appear to occur primarily as an artifact of the two-choice hiding task employed by those researchers. When more than two search alternatives are provided, so that B-trial search errors are not constrained to be returns to the A location, infants do not demonstrate any tendency to search at the A location when erring during B-hiding trials, in direct contradiction of Piaget's action-object account of infant search errors.

\section{General Support for a Memory-Based Explanation of Search Errors}

In contrast, the proposed memory explanation of infant search behavior has been supported by the pattern of search performance observed in the present unconstrained hiding situation. According to this explanation, infants are capable both of understanding fundamental relations of objects and space and of storing in memory at least some information about the current location of an object as it is successively hidden in different spatial locations. Search errors occur because the information encoded and/or retrieved is not always precise enough to denote the specific current location of the object. Thus, when search errors occur, they should typically reflect partial knowledge or retention of the object's current location. In the context of the present situation, errors would be predicted (1) to cluster around the currently correct location on any given trial, forming a spatial gradient stemming outward from this location, and (2) to converge upon the correct location over trials at the same hiding location. Furthermore, any change in the task that places added demands on the infant's ability to process, store, or retrieve information should increase the number of search errors [as, for example, when the object's location is changed from an old (A) to a new (B) location].

Clearly, the pattern of errors obtained in the present two experiments has been consistent with these expectations of the memory explanation. On the A-hiding trials, errors formed a spatial gradient stemming outward from the correct location and converged upon the correct location over hiding trials at that same location. When the object was moved to a new location (B), there was a significant decrease in correct search performance, and the direction of search errors switched to the new location. Then, over trials at the new location, correct performance consistently increased, with search attempts steadily converging upon the new correct location, reaching A-trial levels by the third B trial in Experiment 2. At all times, the direction of search appeared to be influenced by information about the object's current location-not by information about previous action-locations involving the object.

\section{The Lack of Specific Proactive Interference in the Present Five-Choice Tasks: A Problem for the Memory-Based Explanation?}

Two aspects of B-trial performance observed in both of the five-choice tasks of the present two experiments warrant further discussion: (1) the significant drop in performance between the last A-hiding trial and the first B-hiding trial and (2) the poorer performance on the first $B$ trial than on the first A trial.

\section{The First B-Hiding Trial Versus the Last A-Hiding Trial}

Whereas we see the drop in performance on the first $B$ trial to reflect, at least in part, interference effects from the previous $A$ trials on the encoding and retrieval processes occurring at $B$ and, thus, to indicate the operation of a general type of proactive interference, there is 
no evidence in the present results for the operation of specific proactive interference-that is, specific response competition between $A$ and $B$ search responses as measured by intrusions of the $A$ response when the $B$ response is appropriate. Across the 52 first $B$ trials on the five-choice tasks of the present two experiments, 28 errors were made, but only 2 of these, or $7 \%$, were incorrect searches at the A location. By chance alone, one would expect $20 \%$ of the errors to occur at the A location.

Since, on the basis of research investigating memory for verbal material in adults, one might expect some evidence for the operation of specific proactive interference in producing the drop in performance from the last A- to the first B-hiding trial, the lack of such evidence in the present situation could be considered by some to create problems for a memory explanation of infant search behavior. However, we feel that the lack of such specific interference can be accounted for in terms of certain important differences between the present situation and the typical situation in which specific proactive interference is observed. In the present situation, both the spatial nature of the information that needed to be encoded and the nonverbal, or preverbal, nature of the subjects would seem to preclude the use of verbal encodings for this information. Hence, we assume that the present infants encoded the location of the object in spatial rather than verbal terms. In addition, since the physical A and B locations of the present five-choice apparatus were spatially quite distinct, it seems likely that the infant's functional memory codes for the A and B locations in the present situation were quite distinct. Thus, at the time of retrieval, the infant would have had little difficulty in discriminating the memory trace of the B location from that of the A location-a situation in which, drawing from the proactiveinterference literature, one should expect little or no specific proactive interference or response competition between the $\mathrm{A}$ and $\mathrm{B}$ search responses. On the other hand, general interference effects from the just-previous series of A-hiding trials could have made encoding of the new B location more difficult, resulting in an initially poor or insufficient memory code for the B location. However, even when the memory code for the new B location was not sufficient to produce a correct search response or to differentiate the new B location from those alternative hiding locations most spatially similar to the new B location, it most likely still contained enough distinctive spatial information in the present situation to not be confusable with the trace of the spatially dissimilar A location and, thus, still directed the infant's search response to the general vicinity of the correct location. (See Crowder, 1976, pp. 211.216, and Gardiner, Craik, \& Birtwistle, 1972, for a discussion of the role of retrieval discrimination as the basis for specific proactive interference in adult verbal memory studies.)
It is interesting that other studies designed to assess the role of proactive interference in the production of search errors made by another nonverbal organism-the rhesus monkey-have also found little or no evidence for the operation of specific proactive interference. In a study by Medin (1969), rhesus monkeys performed a task quite similar to the one performed by the present infants. The monkeys had to remember, for delay intervals of $0,1,2,5,10$, or $20 \mathrm{sec}$, behind which door in a $4 \times 4$ matrix of doors a food reward had been hidden. If proactive interference or response competition from the just-preceding trial is a principal source of forgetting in this situation, as seems to be the case in adult verbal memory studies (see Crowder, 1976, pp. 203-206, and Fuchs \& Melton, 1974, for a discussion of sources of proactive interference effects), then a frequent type of error should have been searches at the location that had been correct on the just-preceding trial. However, an analysis of the location of incorrect search errors revealed little evidence for such specific proactive errors. In the Medin paradigm, one would expect $6.25 \%$ of the incorrect searches to occur by chance at the location of the previous hiding trial (specific proactive errors). For the two delay intervals most similar to those in the present study, 2 and $5 \mathrm{sec}$, the percentages of errors that were such specific proactive errors were $8.9 \%$ and $7.6 \%$, respectively. In addition, $83 \%$ of all these "specific proactive errors" occurred on trials in which the correct location on that trial happened to be adjacent to the location that had been correct on the preceding trial. Thus, in the Medin study, as in the present studies, the spatial similarity or closeness of an alternative location to the currently correct location of the object appears to have been a primary determiner of whether that location will be the locus of any incorrect search attempts.

In another series of experiments designed to test a proactive-interference analysis of errors, Fletcher, Garske, Barron, and Grogg (1968) found no evidence for the operation of specific proactive interference in producing the search errors of rhesus monkeys in the performance of two-choice hiding tasks. In fact, these authors concluded that even those search errors that appeared to be proactive-interference errors actually occurred as the result, and not the cause, of intratrial factors such as not attending and forgetting.

Our analysis of the drop in correct performance on the first B-hiding trial in the present five-choice situation has somewhat the same flavor as the Fletcher et al. (1968) analysis in that we see the decrement as arising, to a large extent, from general proactive-inteference effects of the previous series of A-hiding trials that can be characterized as changes in the intratrial demand characteristics of the first $B$ trial as compared with those of the last A trial. To illustrate, on all A trials, the infant must notice and encode only that the object is hidden at $\mathrm{A}$. On the first B-hiding trial, the infant must first notice that the object is now being hidden in a 
different location and then come up with an encoding of that new location that will serve to differentiate it from the other possible locations in the present fivechoice tasks, including the old A location. In short, the infant has more information to process on the first $B$ trial than on the last A trial and, consequently, has less time or capacity to come up with a precise encoding of the object's location on the first B-hiding trial. Furthermore, an adequate encoding of the new B location must, in a sense, be more complex than the encoding of the $A$ location, since the B location's encoding must both differentiate it from the other possible hiding locations and also include some sort of temporal or ordinal denotation that allows the infant to know that it is the most recent or last location of the object.

For all these reasons, even though the infant's initial encoding of the new B location would tend to contain some positional or spatial information about the object's new location, this information would frequently not be precise enough to differentiate the new $B$ location from those alternative locations most spatially similar to it. On the other hand, in the context of the present task (for reasons discussed above), such an encoding would tend to not be confusable with the previous encoding of the spatially distinct A location, and, thus, the infant's attempts to retrieve the new B location of the object on the basis of such an encoding would not be seriously hampered by response competition from the memory trace of the previous A location. Hence, although the infant's initial encoding of the new B location would frequently not be sufficient to direct search to the precise new location of the hidden object, it should typically be sufficient to direct search at least to the general vicinity of the new location. (It also seems reasonable that, occasionally, an infant would not be able to encode even imprecise spatial information about the object's new location or, for some reason, not even notice or encode that the object had been moved to a new location on the first $B$ trial. While the present data indicate that such events rarely happen, their occurrence could account for the few A-returning search errors observed in the present studies.) Over repeated trials at the new location, however, the information-processing demands would quickly become more like those of the preceding A trials, the infant's encoding of the new location should become more precise as well as less vulnerable to loss during the retention interval, and performance should rapidly return to A-triai levels.

It is important to point out that, although our analysis of first B-trial performance in the present fivechoice tasks does not attribute the observed decrements to the operation of specific proactive interference or competition between $A$ and $B$ search responses, we do not, on the other hand, rule out specific proactive interference as one possible contributor to the infant's inability to retrieve the object's correct location in some task situations and, would, in fact, expect a greater influence of such specific retrieval competition in cer- tain task situations. For example, if location A were to be made more similar than the other incorrect locations to location B or, if the hiding procedure used were one in which the infant's attention were to be directed to $A$ or cues associated with $A$ were reinstated at or just prior to the time the infant needed to retrieve information about the object's current location (see, for example, Harris, 1973), specific proactive interference or retrieval competition between A and B responses might well act to reduce the infant's ability to retrieve the currently correct location information.

It could also be the case that specific proactive interference plays a larger role in producing forgetting on the first B trial in the typical two-choice task than in the present five-choice tasks, since, in two-choice hiding tasks, the A location is ipso facto the most similar alternative location to the correct B location. Furthermore, there would seem to be a real perceptual sense in which the $\mathrm{A}$ and $\mathrm{B}$ hiding locations of the present two-choice task, as well as those of other two-choice tasks, are more confusable or similar than the A and B hiding locations of the present five-choice tasks. In the latter case, the A and B locations are separated by other intervening locations, which could make their identity as opposite anchor or end locations more perceptually salient or noticeable, affording the infant more differential encoding cues for these locations.

On the basis of this difference between the typical two-choice task and the present five-choice tasks, we see the following tentative picture emerging to relate infant search behavior in the two types of situations. First, in both situations, infants are frequently able to encode and store some information about the object's new spatial location on the first B-hiding trial. However, for all the reasons discussed above, the information encoded is often not precise enough to differentiate the new B location from the location or locations most spatially similar or confusable with it, which, in the five-choice situation, are the alternative locations closest to the new B location and, in the two-choice situation, is the old A location. Thus, it could be that specific proactive interference or competition between $A$ and $B$ responses is largely responsible for the below-chance performance often observed on the first B trial of twochoice tasks, including that on the present two-choice task of Experiment 2, whereas the more generalized proactive-interference effects, discussed above, would be responsible for the drop in B-trial performance on multichoice tasks, such as the present five-choice tasks.

It is important to remember, however, both in the case of the present two-choice task as well as in all previous two-choice tasks, that, on the basis of such tasks alone, one is no more entitled to attribute errors occurring at the A location to specific proactive interference than one is entitled to attribute errors at the A location to perseveration. On the other hand, since a memory explanation is also compatible with the results obtained in the unconstrained, multichoice situations of 
the present two experiments and the perseveration explanation is not, credence is lent to a specific proactiveinterference explanation of A-location errors in the typical two-choice hiding task. In other words, the patterns of results obtained in the five-choice tasks of the present two experiments converge to support a memory explanation of search on two-choice tasks as well as on multichoice tasks.

\section{The First B-Hiding Trial Versus the First A-Hiding Trial}

In both of the present two experiments, as well as in most of the two-choice studies previously reported in the literature, first B-trial performance tends to be poorer than first A-trial performance. We feel that two factors are largely responsible for this common finding. First, the analysis presented above regarding first B-trial versus last A-trial performance also applies to first Atrial versus first B-trial performance. That is, just as the intratrial-demand characteristics of the first $B$ trial are different and greater than those of the last A trial, they are also different and greater than those of the first $A$ trial. On all $\mathrm{A}$ trials, including the first, the infant must notice and encode only that the object is hidden at $\mathbf{A}$. On the first B trial, however, the infant must first notice that the object is being hidden at a new location and then encode that new location in a way that both differentiates it from the other locations and denotes it as the most recent location of the object. In other words, on the first B trial, as opposed to the first A trial, updating demands as well as encoding demands are placed on the infant. Thus, there is more information that must be processed and stored on the first $B$ trial than on the first A trial, and, consequently, the infant's encoding of the object's location on the first B trial should typically be less precise.

A second factor that we feel must contribute to the common finding of better first A-trial than first B-trial performance is the typically employed procedure of conducting warm-up trials in the hiding location that is to be the A location in the experimental trials. Thus, by the time the infants are presented with the first actual A trial, they have already had considerable practice in retrieving the toy from that location, a factor that should, in terms of the present memory explanation, give the infants a considerable encoding advantage on the first $\mathrm{A}$ trial as compared with the first $\mathrm{B}$ trial. There is substantial evidence in both of the present two studies, as well as in others in the literature, that correct search performance increases with repeated hidings of the object at the same location.

In light of the present analysis, one might well ask why we did conduct our warm-up trials in that location that was to become the A location of the experimental trials. We did so because that is the procedure that has been used in most previous two-choice Stage IV studies, and we wished to depart as little as possible from all aspects of previously employed procedures, except for the use of more than two hiding locations. Why previous studies have typically used this procedure is hard to say, but since memory was not considered to be a primary factor in producing infant search errors, the potential memorial consequences of such a procedure would not have been considered.

\section{A Memory-Based Interpretation of Past and Future Results}

Although the present memory explanation accounts for several aspects of the observed search behavior, it does so at a general level and needs to be developed in further detail. At present, the explanation makes two basic contentions: (1) that infants do not suffer from an inability to separate the actual spatial locations of objects from the locations of actions performed upon these objects, and (2) that infants can effectively encode, store, and retrieve information concerning the current spatial locations of objects in their environment. Another way of stating the latter contention is that infants have the ability to update their memories regarding the current location of objects in their environment. Whether infants always make use of this ability and under what circumstances it is more or less effective are, of course, separate issues. Furthermore, the ways in which encoding, storage, and retrieval processes may be different or limited in the infant, as compared with the adult, and what the relative roles of these processes are in producing both successful and incorrect search behavior in the infant remain to be clarified. For example, it may turn out that infants have more trouble with one process than another at different points in their development. Perhaps storage capacity remains relatively constant throughout development and from individual to individual, whereas encoding and retrieval processes are more subject to individual patterns of development through exposure to different types of experiences that lead the infant to develop encoding and retrieval strategies of varying degrees of effectiveness. In addition, we feel that the relative effects of these processes on performance are likely to vary as a function of the nature or demands of the particular task to be performed-for example, the type of cues that are potentially available for encoding the object's location, or the type of distraction or interference occurring between hiding and search.

Some light can already be shed on these issues by examining the findings of previous research in terms of the present memory analysis. For example, several studies (Bremner, 1978a, 1978b; Butterworth, Note 2) have found that in two-choice hiding tasks, search performance is improved when covers of two different colors are used on a hiding apparatus of uniform color, but not when the covers are the same color and each side of the apparatus has a different color. The differential effects of these two stimuli can be explained in terms of their potential to be noticed and used as encod. ing cues by the infant. The cloth covers are picked up and handled by the infants as they perform the search 
task, making these items highly salient stimuli in the infants' immediate surround and, consequently, more likely to be used by the infants as cues for encoding the hidden object's location.

A similar interpretation can be made of Bremner's (1978a, Experiment 2) finding that infants make fewer search errors when the spatial relationship of a hidden object to the infant changes owing to movement of the infant rather than to movement of the object. As Bremner suggested, the movement of the infants could alert them to the fact that a change is taking place. We would further suggest that this movement alerts the infant to the need to encode the object's location in terms of a cue that will remain stable or invariant with respect to rotation. When such a cue is readily available-such as salient black and white covers-the infants' search errors are dramatically reduced. Acredolo and Evans (1980) also showed that 6-, 9-, or 11-month-old infants are better able to keep track of positions in space when landmarks are provided. Similarly, Rieser (1979) demonstrated that 6-month-old infants can encode and remember spatial-location information in terms of gravitational and landmark cues when such cues are made available. These results point to insufficient encoding as the primary source of infant search errors. Note, however, that it is not the case that infants are unable to encode the spatial location of the object effectively; rather, the constraints of the task are usually such that little in the way of good encoding cues are available to the nonverbal infant-that is, external cues about a given location that can be encoded and, when retrieved, can be used by the infant to differentiate that location from another. When such external cues are made available (such as salient black and white covers), the infant can take advantage of these cues to encode more effectively the correct location of the hidden object.

Other studies reported in the literature can also be interpreted as supporting or being consistent with the current account of infant search behavior. For example, Gratch et al. (1974) and Harris (1973) found that, in order to produce search errors during B-hiding trials, it was necessary in the two-choice hiding task to insert a delay between the time the object was hidden and the time the infant was allowed to search for the object. Fox, Kagan, and Weiskopf (1979) also showed a critical effect of delay on the frequency of B-trial errors. Webb, Massar, and Nadolny (1972), using a three-choice hiding paradigm involving 14- and 16-month-old infants, concluded that the high incidence of correct searches obtained when the 16-month-old infants were given a second opportunity to search for objects indicated that at least some information about the object's current location had been stored in memory. In research both with 9-month-old infants performing slightly different three-, five-, and six-choice visible-hiding tasks and with 12-to-14-month-old infants performing a five-choice invisible-displacement task, we have found patterns of search errors essentially identical to the ones observed in the present five-choice visible-displacement tasks. (See Cummings \& Bjork, 1981, 1983b, for further details of this research.)

In other research with older infants, Sophian and Sage (1983) recently examined the performance of 13 . and 21-month-old infants across a series of search tasks, including visible-displacement tasks that utilized three possible hiding locations. In this innovative study, Sophian and Sage found that when infants made errors on the visible-displacement tasks, those errors were just as likely to be at the location in which the object had not been hidden on that trial, designated the control location by Sophian and Sage, as at the location in which the object had first been hidden on that trial (i.e., the A location), designated the relevant-butincorrect location by Sophian and Sage. Although this result is not directly comparable, owing to slight differences in the hiding procedures used by Sophian and Sage, it is certainly concordant with our finding of no tendency on the part of 9-month-old infants to make A-returning search errors when not forced to do so by the constraints of the two-choice hiding paradigm.

Sophian and Sage (1983) interpreted their finding of no difference in the likelihood of errors occurring at the control location versus the relevant-but-incorrect location on visible-displacement problems as indicating an inability or failure on the part of the infants to identify the relevant locations in these tasks, that is, the two locations that were actually involved in the hiding problem on a given trial. Beyond the issue of whether this interpretation entails acceptance of the null hypothesis, we feel that a better and more parsimonious interpretation of this finding is that it reflects a limitation of memory rather than an inability to identify or comprehend which two of the three possible locations were actually involved in the hiding problem presented on any given trial.

Again, our reasons for asserting a memory interpretation of this finding stem from an information-processing analysis of the task situation with which the infants in this study were faced. More specifically, in the Sophian and Sage (1983) study, each infant was presented with five different types of hiding tasks in succession. Furthermore, within each type of hiding task, at least three trials were presented to each infant such that the initial hiding was performed equally often in each of the three possible locations and, if the problem involved a second location, all possible pairings of first and second locations were used equally often. In other words, which location happened to be the control location and which location happened to be the relevant-but-incorrect location changed from trial to trial on the same problem type for each infant. From an information-processing point of view, then, this counterbalancing scheme created a hiding-task situation that would place tremendous updating demands on the infant's memory and one in which potent interference effects would be operating from one trial to the next. Given these up- 
dating demands, trying to keep track of which two locations happened to be relevant on each trial and then choosing between them would be a less efficient and more demanding strategy in terms of the memory load it would place on the infant than would simply trying to note and remember in which location the object was ultimately left and then searching there, which, in fact, is the strategy that Sophian and Sage suggested the infants in their study seemed to have adopted. It is important to note, however, that adoption of such a strategy does not imply that the infants were unable to identify the two relevant locations on each trial. Thus, while Sophian and Sage's interpretation is not inconsistent with the data, we feel that it goes beyond what is called for by the data and that the present memory interpretation is a more parsimonious interpretation of their finding.

Another point we would like to make with respect to the Sophian and Sage (1983) study relates to the concern these authors expressed with respect to their finding of a relatively low performance level on even their simple single-hiding problem, the task in which the object was hidden in only one location and then the infant was allowed to search for it. As Sophian and Sage pointed out, the average performance level of their 13-month-old infants on this task was considerably poorer (56\% correct) than the performance level of 9-month-old infants on similar problems, for which they gave as an example the A-hiding trials in previous twochoice tasks. As Sophian and Sage suggested, one reason for the lower performance level in their study may have been the use of a three-alternative hiding task. Although possibly a contributing factor, the average performance levels of the 9-month-old infants on the A trials of the present five-choice hiding tasks, which were $86 \%$ and 73\% correct in Experiments 1 and 2, respectively, argue that the presence of three alternatives could not be the entire reason for the poor performance of Sophian and Sage's 13-month-old infants. Instead, we feel that their procedure of changing the position of the correct or ultimate hiding location of the object on each successive trial of a given problem type was probably the major reason for this finding.

To be more specific, we suspect that this procedure created, in essence, an analog to the Brown-Peterson task of the adult verbal memory literature (Brown, 1958; Peterson \& Peterson, 1959). In a Brown-Peterson task, in which subjects are asked to remember similar types of items on each trial (say, three consonants), recall performance tends to be perfect on Trial 1 and then drops off rapidly, reaching asymptotic performance level over three to six trials (e.g., Fuchs \& Melton, 1974; Keppel \& Underwood, 1962; Loess, 1964). Furthermore, if after asymptotic performance level has been reached, the type of items to be remembered is changed (say, from consonants to digits), recall performance tends to recover (Wickens, Born, \& Allen, 1963). Thus, our conjecture that Sophian and Sage's (1983) pro- cedure created an analog to the Brown-Peterson task implies that the low performance level they observed on the single-hiding problem could be due to the fact that this performance level was arrived at by averaging across the three trials presented to each subject. Although Sophian and Sage did not present single-trial performance data, we suspect that first-trial performance would be higher and more similar to previously reported performance levels than that obtained by averaging across the three trials. Furthermore, Sophian and Sage's procedure of switching from one type of hiding container to another between problem types could be analogous to switching from one type of material to another in the adult task, thus allowing performance levels to recover, which, in turn, would account for why no effect of the order of problem type was obtained and why the overall level of performance based on data only from the first problem type that each infant received was about the same as the overall level of performance based on all the data.

Although Sophian and Sage (1983) were rightfully concerned with the question of whether their method of testing the same infant across a series of different problem types might raise problems of carry-over effects from one problem type to another, their analyses ignored the possibility of carry-over effects from one trial to the next within a given problem type. From the perspective outlined above, however, memorial intertrial carry-over effects were probably potent and cannot be ignored if a proper understanding of infant search behavior in such tasks is to be understood. Our point here, and a recurring thesis throughout the present paper, is that without subjecting the various tasks we use in infant research to an information-processing analysis that, among other considerations, attempts to recognize the various demands such tasks place on the infant's memory, we can easily be misled in our interpretation of the infant's behavior on such tasks.

In addition to the supportive evidence from the studies discussed above, the plausibility of a memory explanation for infant search behavior is enhanced by recent evidence (Fox et al., 1979; Kagan \& Hamburg, 1981) of significant increases in memory-related functions in the last half of the 1st year of life-a period in which the infant's performance on object-permanence tasks also rapidly improves (Gratch \& Landers, 1971). Finally, in a recent discussion of the development of memory in infancy, Schacter and Moscovitch (in press) also argued compellingly for a major role of memory in producing the traditional $\mathrm{A} \overline{\mathrm{B}}$ error.

On the other hand, some results previously obtained in two-choice hiding tasks seem to be at odds with the present memory explanation of infant search behavior. In particular, Butterworth (1977) and Harris (1974) reported that infants err even when the object is visible during B-hiding trials - -a finding that, on the surface, at least, appears to be inconsistent with the notion that B-trial errors are primarily produced by memory failures. 
In Harris's (1974) experiment, infants first received three pretest A trials in which a car, located behind a transparent barrier, was pushed down a track to one of two transparent doors, which the infants could open to retrieve the car. Infants then received, in counterbalanced order, one A trial, in which the car was pushed to the same door as before, and one B trial, in which the car was pushed to the opposite door, but now both doors were locked. On both A and B trials, most infants first approached the door behind which the car was visible, but, on finding it locked, then approached the opposite or empty door; thereafter, they vacillated between the two locked doors. Because infants approached the visibly empty A door on B-test trials, Harris ruled out a memory explanation of the $\mathrm{A} \overline{\mathrm{B}}$ error. However, this interpretation does not take into account two important aspects of the infants' behavior. First, on B test trials, most infants first approached the door behind which they could see the car (i.e., the B door) and, only after finding it locked, did they then approach the visibly empty A door. Second, and most tellingly, on A test trials, infants also approached the visibly empty B door after finding the A door locked. That is, approaching the empty door was not peculiar to B test trials. Thus, a more plausible interpretation, which would be consistent with both A- and B-trial performance, is that infants saw and comprehended where the car was on both types of trials and were merely trying all possible strategies to gain access to it. Hence, we feel that Harris's results are more reasonably attributed to problemsolving strategies and are largely irrelevant to a memory explanation of infant search errors.

In Butterworth's (1977) experiment, infants were tested in three types of two-choice hiding tasks: (1) the standard Stage IV task, in which the object was completely hidden at $A$ and $B(O H) ;(2)$ one in which the object was covered by a transparent cover at $\mathrm{A}$ and $\mathrm{B}$ (OC); or (3) one in which the object was not covered at $A$ and $B(O V)$. In all three conditions, infants made more errors on the first B-hiding trial than on the first A trial, which in the $\mathrm{OC}$ and $\mathrm{OV}$ conditions might be considered as being inconsistent with a memory explanation of infant search errors. However, in all three conditions, B trials presented infants with a completely different problem from that presented on previous $\mathrm{A}$ trials, due to the introduction of a 3-sec delay between hiding and search. During this delay, the sudden introduction of which was probably frustrating or at least distracting to the infants, it is quite possible that some infants looked away from B after the object was hidden and then failed to look back at the time of search. Moreover, consistent with a memory explanation of infant search errors, infants in the OV condition were generally correct, whereas performance in the $\mathrm{OH}$ condition was significantly worse (chance level). On the other hand, Butterworth's finding of no significant difference in the number of errors made in the $\mathrm{OH}$ and $\mathrm{OC}$ conditions is puzzling. If the object was still com- pletely visible when covered by the transparent Perspex covers used in the $O C$ condition, then one would expect performance in this condition to be essentially like that in the OV condition. It may be that the act of lowering the transparent cover over the object was distracting in some way, thus increasing the likelihood that infants would look away from B after the object was hidden and then fail to look back at the time of search. Another possibility is that, except from a certain vantage point that was not assumed by all infants at the time of search, the toy was not clearly visible through the transparent cover. Clearly, further research is needed to determine the source of difficulty in this condition.

A recent experiment by Willatts (1979) might also seem to pose a problem for a memory explanation of infant search errors. In Willatts's experiment, 4- and 5-month-old infants were given five 20-sec trials in which they could reach for an object placed to either the left or right of their midline (the A trials). Then, for half of the infants in each age group (the experimental groups), the object was moved to the opposite side of the midline (i.e., a B trial), and for the other half (the control groups), the object remained in the same place (i.e., another A trial). Willatts took two behavioral measures: (1) manipulation of the object or moving of the infant's hand through the region of space previously occupied by the object and (2) fixation of the object or of the location in which the object had previously been located. Willatts found no differences in these measures between the 5-month-old experimental and control groups, but the 4-month-old experimental group reached more into the empty place (the spot in which the object had been on the previous A trials) than did the same-age control group (the corresponding spot on the opposite side for them) and fixated the empty spot more than did the controls. However, the 4-month-old experimental group also fixated the object far more than they fixated the empty place (i.e., the previous A location). In fact, of the $20 \mathrm{sec}$ of observation time, the experimental infants fixated the empty place for an average of only $1.4 \mathrm{sec}$, which was possibly about the amount of time that would be necessary to look at that spot and determine that the object was no longer there or even perhaps that there was not another object there as well. On the other hand, the 4-month-old experimental infants spent less time, overall, reaching than did their controls and spent as much time reaching into the empty place as they did reaching into the place with the object. Thus, although the 4-month-olds were able to identify the object's new position visually, they did not seem able to use this information to control their reaching behavior. Such reaching errors had disappeared in the 5-month-olds. As Willatts suggested, this disappearance in reaching errors could occur because 5-month-old infants have learned that an object can exist in a variety of places, or it could occur because the infants have improved in their reaching skills. Perhaps the 4-monthold infants are unable to inhibit a previously successful 
action or cannot rapidly substitute one reach for another. Thus, although the infant is fixating the new location of the object, it cannot inhibit the reaching response that previously brought the object into contact with its hands. Indeed, the fact that the 4-month-old experimental infants spent less time, overall, reaching than did their corresponding controls might be an indication of some sort of interference or mutual inhibition between the two reaching responses. Although Willatts stated that his findings did not indicate whether the object-concept or the motor-skills explanation was appropriate, we would argue that only the motor-skills explanation is consistent with both the fixation and the reaching data.

Using a paradigm in which different types of intervening tasks were introduced during a 90-sec interval between A- and B-hiding trials, Frye (1980) evaluated various explanations of the $A \bar{B}$ errors. To test Harris's (1973) proactive-interference explanation of $A \bar{B}$ errors, Frye compared B-trial performance when this interval was filled with a distracting activity with performance in a no-activity (control) condition, arguing that proactive interference should be reduced in the distraction condition compared with that in the control condition, and that, thus, if proactive interference produces $A \bar{B}$ errors, there should be fewer search errors in the former condition. Frye found no difference in first-trial B performance between the distraction and control conditions and, thus, argued against a proactive-interference explanation of $\mathrm{A} \overline{\mathrm{B}}$ errors. However, as argued by Schacter and Moscovitch (in press), and as it also appears to us, this finding is at best inconclusive with respect to the role of proactive interference in producing search errors, since, as described by Frye, there seems to have been little difference in the actual activities taking place during the 90-sec interval between the $\mathrm{A}$ and $\mathrm{B}$ trials in these two conditions. Although infants in the distraction condition were given a doll with which to play during this interval and infants in the control condition were not, Frye reported that if infants in the control condition became restless or bored, which in a $90-\mathrm{sec}$ interval probably happened frequently, the experimenter made a special effort to engage the infant in play-an activity that would seem to be equally as distracting to the infant as, if not more so than, playing with a doll.

\section{Infant Memory and Egocentrism}

The present results and memory-based explanation also have important implications for recent accounts of infant search behavior in terms of egocentrism. Several investigators (Acredolo \& Evans, 1980; Bremner, 1978b; Butterworth, 1977) have explained infant search behavior by suggesting that infants use either an objective or an egocentric frame of reference to guide search. The infant's selection of spatial location codes is thought to depend, in part, upon the characteristics of the task situation, including the nature of the spatial-location cues available. Whereas the notion that task demands and the availability of different types of spatial-location cues should affect the infant's search performance is consistent with the present memory account, our position with respect to the infant's use of egocentric versus objective or allocentric cues to encode spatial information is somewhat different from that of these researchers, which seems to be that the infant's predominant means of conceptualizing space are egocentric in nature and that encoding spatial information in terms of egocentric codes is a basic tendency that can be overridden or suppressed only in certain situations. In contrast, we feel more comfortable with the notion that egocentric codes are but one of several types of codes available to the infant for processing and encoding spatial information. Furthermore, rather than viewing egocentric encoding as a preferred or dominant method of encoding information, we see it as being more like a strategy of last resort. That is, we feel that egocentric codes would tend to be used or relied upon only in the absence or unavailability to the infant of other salient and more reliable objective cues for processing and encoding spatial information.

In addition, although we feel that egocentric cues can certainly be used by the infant and probably are used both by the infant and by the adult individual in certain situations, we do not feel that it is necessary to draw upon the concept of egocentrism, or the notion that the infant's predominant means of conceptualizing space are egocentric, in order to account for infant search errors. The primary basis for our position is that there is little compelling evidence that the infant's predominant mode of search is egocentric. Two-choice tasks artifactually constrain all infant search errors to be "egocentric." In the unconstrained tasks used in the present research, infants gave no evidence of employing egocentric spatial-location codes. Furthermore, in one of the few studies to address the issue of egocentric encoding and also to employ more than a two-choice task, Rieser (1979) obtained results indicating that even 6-month-old infants can encode and remember spatial information in terms of geocentric and landmark cues when such cues are made available.

Rieser's (1979) procedure in this study was to first train 6-month-old infants to look toward one of four possible doors for a visual reward when they were cued by a signal bell. After reaching a specified learning criterion, infants were tested in one of six conditions. In each testing condition, the infant was first passively rotated $90 \mathrm{deg}$; what varied in each condition was the type of information available for keeping track of the location of the previously rewarded door. When only minimal, passive-movement information could be used to keep track of the previously rewarded door's location, the infants looked predominantly at the egocentric door. However, when gravitational information was available for encoding and keeping track of the previously rewarded door's location in geocentric terms, infants looked predominantly at the geocentric (or 
correct) door. From the pattern of results obtained across conditions, Rieser concluded that 6-month-old infants can encode and remember spatial information in terms of egocentric, geocentric, or landmark cues but that the egocentric code exerts a stronger influence on the 6-month-old infant's visual search than does either of the other types of codes.

Although we agree with the first part of this conclusion, we feel that the second part is not yet warranted. First, the latter part of this conclusion seems not to give enough weight to the finding that in the condition in which gravitational information was available for encoding the rewarded door's location, the infants looked predominantly at the geocentric door. In other words, in the condition in which salient information was available for encoding the location of the correct door in geocentric terms, the infants' visual search was guided more by the geocentric code than by the egocentric code. Second, Rieser (1979) seemed largely to come to the latter part of the conclusion above because, in the condition in which the geocentric and egocentric doors were both marked by patterns (Condition E-G patterned), ${ }^{2}$ infants looked more at the egocentric door, whereas in the condition in which the geocentric and an irrelevant door were both patterned (Condition I-G patterned), infants looked more at the geocentric door. To Rieser, this pattern of results indicated that the egocentric code is stronger than the geocentric code in guiding visual search. However, from an informationprocessing point of view, a comparison of these conditions did not constitute an adequate test of the primacy of the egocentric code. In the E-G patterned condition, the egocentric door was not only patterned similarly to the geocentric door, but was also spatially adjacent to it, whereas in the I-G patterned condition, the irrelvant door, which was patterned similarly to the geocentric door, was also the door most spatially distant from the geocentric door. Thus, in the former condition, the egocentric door was confusable with the geocentric door in two ways, whereas in the latter condition, the irrelevant door was confusable with the geocentric door in only one way. In other words, a spatial-adjacency or spatialsimilarity variable had been confounded with the egocentric/irrelevant-door variable in these two conditions. Given this problem and the finding of the present two studies of a major role of spatial adjacency or similarity in determining the locus of search errors, it seems best to reserve judgment as to the relative potency of egocentric and geocentric codes in guiding the 6month-old infant's visual search. At present, we see Rieser's results as being compatible with the notion that infants tend to rely on egocentric codes to remember spatial information only when other salient and more reliable cues are not available for encoding and remembering such information.

In another study investigating whether infants code spatial-location information in geographic or egocentric terms, Wishart and Bower (1982) used a procedure somewhat similar to Bremner's (1978a) and Rieser's (1979), in which, after an infant watched as an object was hidden in one of two or one of three containers sitting on a table, either the infant or the table, or both, were rotated, and the infant's task was to keep track of the location of the container in which the object had been hidden. Three groups of infants were tested: a cross-sectional group ( 12 to 24 months), a longitudinal group (12 to 24 months), and an accelerated group ( 8 to 20 months). Whereas the results from Wishart and Bower's two-container task suffer from the interpretation problems of all two-choice search tasks and, thus, do not shed any light on the question of the prevalence of egocentric errors in infant search behavior, the results of their three-container task are not similarly confounded. With respect to this point, it is interesting to note that, although on the one hand, Wishart and Bower themselves argued that the results of their twocontainer series were largely uninterpretable, since, without a viable "other" response, any search response must have been either egocentric or geographic, they then do not fully make use of this observation in interpreting the results of their three-container series. To illustrate, in all three groups of infants, the geographic response was by far the most predominant response and, in two of the groups (longitudinal and accelerated), the frequencies of the "egocentric" and "other" errors were essentially equal. Despite this finding, Wishart and Bower concluded that egocentric responding continues well into the second half of the 2 nd year of life. In other words, they seemed to ignore the message yielded by the pattern of search with respect to "other" responses in interpreting their results. One could equally well argue that the infants in these two groups either remembered the location of the correct container or guessed, choosing randomly between the two other containers. Furthermore, on the basis of these results, one would be as justified to conclude that "other" responding continues well into the 2 nd year of life as to conclude that "egocentric" responding does. Given no evidence of a tendency on the part of these infants to search at the "egocentric" location rather than at the "other" location, there is no basis for assuming that infants have a tendency to respond egocentrically.

Furthermore, in the one condition in which infants appear to have been making slightly more "egocentric" errors than "other" errors at several of the testing ages (the cross-sectional condition), there may have been a biasing effect operating to produce more responses that would be labeled by Wishart and Bower (1982) as "egocentric" than ones that would be labeled as "other." From Wishart and Bower's description of the threecontainer series, it would seem that on 8 of the 12 trials presented to each infant, the object could be found in either the near or the far center position after rotation. However, it also appears that the "other" container never ended up in the center position after rotation, whereas the "egocentric" container was in the 
center position on 4 of the 12 trials. Thus, on trials in which the infant had lost track of the correct container's location, the infant might be biased toward searching in the near or far center position, which would spuriously produce more "egocentric" responses than "other" responses. Since this bias would only come into play on trials in which the infant had forgotten the correct location, its effects would be more prevalent in the cross-sectional group of infants than in the other two groups and, thus, could have contributed to what appears to be a slight prevalence of "egocentric" responses over "other" responses in that group. In any event, although this nonequivalence in the possible locations of the "correct," "egocentric," and "other" containers presents an interpretation problem for all three groups of subjects, the obtained pattern of results can be accounted for by the information-processing/ memory explanation of search behavior that we are advocating without resorting to the concept of egocentrism. Furthermore, an egocentric explanation seems untenable, as well as unnecessary. Two of the three groups of infants gave no evidence of egocentric responding, and the apparent "egocentric" responding of the third is largely uninterpretable because of the biasing problem discussed above. In addition, even without this interpretation problem, an egocentric account would still be faced with the difficult task of explaining why only one of the three groups of infants showed any preference for egocentric responding.

Thus, although we applaud Wishart and Bower's (1982) use of a three-choice task and find their discussion of the interpretation problems presented by all two-choice studies to agree with the argument that we are making here and have made elsewhere (e.g., Cummings \& Bjork, 1981, 1983; Bjork \& Cummings, Note 1), we see little or no compelling evidence in their study to support the contention that egocentric responding persists well into the second half of the 2 nd year of life or that the infant tends to make spatial judgments or encode spatial information on the basis of self-referents.

In conclusion, we feel that infant search behavior can be explained without appeal to the notion that the infant's predominant means of conceptualizing space are those of egocentrism. Although egocentric codes are certainly available to the infant, as they are, for that matter, to the adult, we see little convincing evidence in the literature that they are the infant's preferred or dominant means of encoding and remembering spatial information. Our position is that the findings of studies that have been interpreted as supporting the infant's egocentric conception of space can be better and more parsimoniously accounted for in terms of an informationprocessing analysis of the task and of the demands the task places on the infant's ability to process, store, and retrieve spatial information. Finally, it should be noted that other researchers are questioning the usefulness of egocentrism as an explanatory concept (see, e.g., Cox, 1980).

\section{A Memory-Based Interpretation of Other Search Errors}

The present memory explanation for the search behavior of 8-to-12-month-old infants gains further credence when one considers the ability of such a memory model to account for search errors assumed to be characteristic of other stages in Piaget's (1954) sensorimotor period. In Stage I (birth to 1 month) and Stage II (1 to 4 months), infants fail to search for an object at all when it is hidden from view. Piaget attributed this behavior to the infant's egocentric concern for his or her own actions without regard to objects. We feel that this behavior would be more parsimoniously described in terms of the current memory analysis as a reflection of extremely limited encoding skills and/or memory storage in young infants.

Piaget (1954) suggested that, in Stage III (4 to 8 months), objects have permanence for the infant only as an extension of the immediate action in progress. Thus, for example, the infant at this stage is able to find a partially covered but not a fully covered object because "the child sees a fragment of the object and the action of grasping thus set in motion bestows a totality on the thing perceived" (Piaget, 1954, p. 35). Again, it seems to us that a simpler explanation of this behavior is to assume that infants are able to find the partially covered but not the totally covered object because, in the former situation, they are provided with a memory aid or retrieval cue (i.e., the visible part of the object) for the object's current location. Visual-tracking studies (e.g., Bower, 1974) have also been interpreted as providing support for a memory, as opposed to an objectconcept, explanation for infant search behavior in this period, but the evidence is ambiguous (Meicler \& Gratch, 1980), and it may be that the visual-tracking paradigm is too fraught with methodological problems to address adequately this issue (Muller \& Aslin, 1978).

During Stage V (12 to 18 months), infants find a directly hidden object (visible displacement) easily, but they have difficulty when the object is first concealed inside a larger object or a container before it is hidden (invisible displacement). According to Piaget (1954), the infant's difficulty in the latter situation arises because the invisible-displacement task causes "the habits of preceding stages to reappear through temporal displacement" (Piaget, 1954, p. 66). In contrast, the proposed memory model would expect the infant to make more search errors in the invisible-displacement task because of the greater load such a task places on the infant's memory in comparison with that of the visible. displacement task. In the invisible-displacement task, the infant must first notice and remember that the toy has been concealed in another, larger container and then the infant must notice and remember the location to which this larger container is moved.

Furthermore, the invisible-displacement procedure can be thought of as functioning essentially like a dis- 
tractor activity in a memory or recall task. That is, before searching for the hidden object, the infant must somehow realize that the object is no longer concealed in the larger container. In the typical invisibledisplacement task, the infant must search the larger container to know that the object has been removed from it. Thus, the task demands of the invisible-displacement procedure both increase the processing and memory load on the infant and require the infant to engage in a distracting activity just prior to retrieving information concerning the most likely last location of the hidden object. These changes in task demands should increase the difficulty of encoding, retaining, and/or retrieving precise location information, and, according to the memory explanation, more search errors should occur. However, the predicted search errors would not be of the type predicted by Piaget's (1954) actionobject account, which assumes that Stage V infants faced with the invisible-displacement task will revert to behavior appropriate to an earlier stage of object-concept development; namely, they will make $A \bar{B}$, or Stage IV, errors. In contrast, the current memory account would expect the increased search errors to reflect partial knowledge of the object's likely new spatial location.

It remains, of course, to substantiate or insubstantiate the validity of the above memory explanations of infant search errors. However, we feel that conceiving of the infant as a less mature or less effective processor of information than the adult individual is a promising theoretical framework, one that is a formidable alternative to the more radical Piagetian view of the infant as a being with concepts that are fundamentally different from those of the adult. In addition, a demonstration that the "object permanence" errors made throughout the sensorimotor period arise from memory limitations or failures without regard to the infant's ability to separate object from action would have profound repercussions for Piaget's (1954) account of cognitive development, since a cornerstone of Piaget's theory is the notion that infants are initially egocentrically concerned only with their own actions and only gradually come to appreciate the significance of objects distinct from action.

\section{CONCLUSION}

In conclusion, the present research has provided a needed and critical test of perseverative search in infant behavior and has shown that the $\mathrm{A} \overline{\mathrm{B}}$ search error held to be characteristic of 8-to-12-month-old infants and indicative of Stage IV in Piaget's (1954) sensorimotor period of development occurs primarily as an artifact of the two-choice search tasks employed by past researchers. Consequently, the considerable theoretical significance that has been attached to the occurrence of the $A \bar{B}$ error is subject to serious question and needs to be reexamined in the light of the present findings.
More specifically, the patterns of search errors obtained in the present unconstrained five-choice search tasks, on both single A and B trials as well as across trials, clearly indicate that infants between the approximate ages of 8.5 and 10.5 months are capable of storing in memory some information concerning the current location of an object as it is hidden in successive spatial locations. There is no evidence that infants revert, owing to a failure to assimilate information about a new hiding location of an object, to the first and/or previous location in which they acted upon the object. At least by the age of 9 months, then, infants appear to appreciate the substantive permanence of objects and the reality of distinct positions in space.

Finally, the failure of Piaget's (1954) action-object theory to account for search errors obtained in an unconstrained situation, combined with the success of the proposed memory explanation to predict the pattern of search errors obtained in such a situation, implies that a memory model, such as the one outlined in the present paper, might better account for search errors made throughout the sensorimotor period. The development of such a model would take a far less radical view of the infant than the one suggested by Piaget, being guided by a conception of the infant as simply a more limited or less efficient processor of information than the adult individual.

\section{REFERENCE NOTES}

1. Bjork, E. L., \& Cummings, E. M. The " $A$, not $B$ " search error in Piaget's theory of object permanence: Fact or artifact? Paper presented at the meeting of the Psychonomic Society, Phoenix, Arizona, November 1979.

2. Butterworth, G. Logical competence in infancy: Object permanence or object concept? Paper presented at the meeting of the Society for Research in Child Development, San Francisco, March 1979.

3. Cummings, E. M., \& Bjork, E. L. Piaget's Stage IV object concept error: Evidence of perceptual confusion, state change, or failure to assimilate? Paper presented at the meeting of the Western Psychological Association, Seattle, April 1977.

\section{REFERENCES}

Acredolo, L. P., \& Evans, D. Developmental changes in the effects of landmarks on infant spatial behavior. Developmental Psychology, 1980, 16, 312-318.

Bower, T. G. R. Development in infancy. San Francisco: Freeman, 1974.

BREMNER, J. G. Egocentric versus allocentric spatial coding in nine-month-old infants: Factors influencing the choice of code. Developmental Psychology, 1978, 14, 346-355. (a)

Bremner, J. G. Spatial errors made by infants: Inadequate spatial cues or evidence of egocentrism? British Journal of Psychology, 1978, 69, 77-84. (b)

Bremner, J. G., \& Bryant, P. E. Place versus response as the basis of spatial errors made by young infants. Journal of Experimental Child Psychology, 1977, 23, 162-177.

Brown, J. Some tests of the decay theory of immediate memory. Quarterly Journal of Experimental Psychology, 1958, 10, 12-21.

BUTTE RWORTH, G. Object identity in infants: The interaction of 
spatial location codes in determining search errors. Child Development, 1975, 46, 866-870.

Butte RWORTh, G. Asymmetrical search errors in infancy. Child Development, 1976, 47, 864-867.

Buttenworth, G. Object disappearance and error in Piaget's Stage IV task. Journal of Experimental Child Psychology, 1977, 23, 391-401.

Corter, C. M., Zucker, K. J., \& Galligan, R. F. Patterns in the infant's search for mother during brief separation. Developmental Psychology, 1980, 16, 62-69.

Cox, M. V. (Ed.) Are young children egocentric? New York: St. Martins Press, 1980.

Crowder, R. G. Principles of learning and memory. Hillsdale, N.J: Erlbaum, 1976.

Cummings, E. M., \& BJonk, E. L. The search behavior of 12 to 14 month-old infants on a five-choice invisible displacement hiding task. Infant Behavior and Development, 1981, 4, 47-60.

Cummings, E. M., \& Buork, E. L. Perseveration and search on a five-choice visible displacement hiding task. Journal of Genetic Psychology, 1983, 142, 283-291.

Cummings, E. M., \& Bjonk, E. L. Search behavior on multichoice hiding tasks: Evidence for an objective conception of space in infancy. International Journal of Behavioral Development, $1983 \mathrm{~b}, 6,71-87$.

Evans, W. F., \& Gratch, G. The Stage IV error in Piaget's theory of object concept development: Difficulties in object conceptualization or spatial localization? Child Development, $1972,43,682-688$.

Fletcher, H. J., Garske, J. P., Barron, T., Grogg, T. M. Intertrial and intratrial determinants of delayed responses of monkeys. Journal of Comparative and Physiological Psychology, $1968,65,66-71$.

Fox, N., Kagan, J., \& Weiskopf, S. The growth of memory during infancy. Genetic Psychology Monographs, 1979, 99, 91-130.

Frye, D. Stages of development: The Stage IV error. Infant Behavior and Development, 1980, 3, 115-126.

Fuchs, A. F., \& MElton, A. W. Effects of frequency of presentation and stimulus length on retention in the Brown-Peterson paradigm. Journal of Experimental Psychology, 1974, 103, 629-637.

Gardiner, J. M., Craik, F. I. M., \& Birtwistle, J. Retrieval cues and release from proactive inhibition. Journal of Verbal Learning and Verbal Behavior, 1972, 11, 778-783.

Gratch, G., Appel, K. J., Evans, W. F., LeCompte, G. K., \& Wright, N. A. Piaget's Stage IV object concept error: Evidence of forgetting or object conception? Child Development, 1974, 45, 71-77.

Gratch, G., \& Landers, W. F. Stage IV of Piaget's theory of infant's object concepts: A longitudinal study. Child Development, 1971, 42, 359-372.

Harris, D. L. Perseverative errors in search by young children. Child Development, 1973, 44, 28-33.

Harris, D. L. Perseverative search at a visibly empty place by young infants. Journal of Experimental Child Psychology, 1974, 18, 535-542.

Jackson, E., Campos, J. J., \& Fischer, K. W. The question of decalage between object permanence and person permanence. Developmental Psychology, 1978, 1, 1-10.

KePPEL, G., \& Underwood, B. J. Proactive inhibition in short- term retention of single items. Journal of Verbal Learning and Verbal Behavior, 1962, 1, 153-161.

Kagan, J., \& Hamburg, M. The enhancement of memory in the first year. Journal of Genetic Psychology, 1981, 138, 3-14.

Loess, H. Proactive inhibition in short-term memory. Journal of Verbal Learning and Verbal Behavior, 1964, 3, 362-368.

Medin, D. L. Form perception and pattern reproduction by monkeys. Journal of Comparative and Physiological Psychology, $1969,68,412-419$.

Meicler, M., \& Gratch, G. Do 5-month-olds show object conception in Piaget's sense? Infant Behavior and Development, 1980, 3, 265-282.

Muller, A. A., \& Aslin, R. N. Visual tracking as an index of the object concept. Infant Behavior and Development, 1978, 1, 309-319.

Peterson, L. R., \& Peterson, M. J. Short-term retention of individual verbal items. Journal of Experimental Psychology, 1959, 58, 193-198.

Piaget, J. The construction of reality in the child. New York: Basic, 1954

RIEsER, J. J. Spatial orientation of six-month-old infants. Child Development, 1979, 50, 1078-1087.

Schacter, D. L., \& Moscovitch, M. Infants, amnesics, and dissociable memory systems. In M. Moscovitch (Ed.), Infant memory. New York: Plenum Press, in press.

Schuberth, R. E., Werner, J. S., \& LipgitT, L. P. The Stage IV error in Piaget's theory of object concept development: $\mathbf{A}$ reconsideration of the spatial localization hypothesis. Child Development, 1978, 49, 744-748.

SOPHiAn, C., \& SAGE, S. Developments in infants' search for displaced objects. Journal of Experimental Child Psychology, 1983, $35,143-160$

Webb, R. A., Massar, B., \& Nadolny, T. Information and strategy in the young child's search for hidden objects. Child Development, 1972, 43, 91-104.

Wickens, D. D., Born, D. G., \& Allen, C. K. Proactive inhibition and item similarity in short-term memory. Journal of Verbal Learning and Verbal Behavior, 1963, 2, 440-445.

Wrllatts, $P$. Adjustment of reaching to change in object position by young infants. Child Development, 1979, 50, 911-913.

WisharT, J. G., \& Bower, T. G. R. The development of spatial understanding in infancy. Journal of Experimental Child Psychology, 1982, 33, 363-385.

\section{NOTES}

1. Since aspects of the present research were first presented (Bjork \& Cummings, Note 1; Cummings \& Bjork, Note 2), several studies not directed at assessing the reliability of the Stage IV error but employing more than two locations in various types of search tasks have appeared in the literature (Rieser, 1979; Sophian \& Sage, 1983; Wishart \& Bower, 1982). These studies are discussed further in the General Discussion section of the present paper.

2. There is a discrepancy between the textual and schematic (Figure 1) descriptions of Condition E-G patterned in Rieser (1979). The present discussion assumes the textual description to be correct and is consistent with it.

(Manuscript received July 23, 1982; revision accepted for publication September 13,1983 .) 